\title{
Computationally Efficient Scale Covariant Time-Frequency Distributions
}

\author{
Selin Aviyente \\ Department of Electrical and Computer Engineering, Michigan State University, East Lansing, MI 48824, USA \\ Correspondence should be addressed to Selin Aviyente, aviyente@egr.msu.edu
}

Received 3 October 2008; Revised 14 January 2009; Accepted 3 February 2009

Recommended by Ulrich Heute

Scale is a physical attribute of a signal which occurs in many natural settings. Time-frequency distributions (TFDs) belonging to Cohen's class are invariant to time and frequency shifts, but are not necessarily covariant to the time scalings of the signal. Conditions on the time-frequency kernel for yielding a scale covariant distribution have been previously derived (Cohen, 1995) . In this paper, a new class of computationally efficient scale covariant distributions is introduced. These distributions are constructed using the eigendecomposition of time-frequency kernels (Burrus et al., 1997). The performance of this new class of distributions is illustrated with examples and is compared to conventional scale covariant distributions.

Copyright ( 2009 Selin Aviyente. This is an open access article distributed under the Creative Commons Attribution License, which permits unrestricted use, distribution, and reproduction in any medium, provided the original work is properly cited.

\section{Introduction}

Scale is a physical attribute of signals just like frequency. Self-scaling of signals is a phenomenon that is observed in different natural settings including biological and acoustic signals. Time-frequency distributions are designed to represent the energy distribution of nonstationary signals simultaneously in time and frequency but do not necessarily reflect the changes in scale. A bilinear continuous timefrequency distribution (TFD) belonging to Cohen's class is represented as follows [1] (all integrals are from $-\infty$ to $\infty$ unless otherwise specified):

$$
\begin{gathered}
C(t, \omega)=\iiint \phi(\theta, \tau) s\left(u+\frac{\tau}{2}\right) s^{*}\left(u-\frac{\tau}{2}\right) \\
\times e^{j(\theta u-\theta t-\tau \omega)} d u d \theta d \tau,
\end{gathered}
$$

where $s$ is the signal, and $\phi(\theta, \tau)$ is the kernel function in the ambiguity domain. For a bilinear time-frequency distribution, scale covariance implies that when the signal is scaled in time, the TFD scales accordingly both in time and frequency, that is, if $s(t) \rightarrow C(t, \omega)$, then $\sqrt{a} s(a t) \rightarrow$ $C(a t, \omega / a)$. For bilinear distributions belonging to Cohen's class, it has been shown that scale covariance is satisfied when the kernel is a product kernel, that is, $\phi(\theta, \tau)=\phi(\theta \tau)$.
There have been various time-frequency representations designed to satisfy scale covariance such as the wavelet transform, the affine class, and the hyperbolic class of time-frequency distributions (e.g., [2-4]). These transforms achieve scale covariance at the expense of losing some desired properties such as time-frequency shift invariance and constant-bandwidth resolution.

In this paper, the focus is on the scale covariance of Cohen's class of distributions. Recent research results in the decomposition of time-frequency kernels for fast computation of TFDs $[5,6]$ will be used to construct a new class of computationally efficient scale covariant timefrequency kernels. It will be shown that it is possible to construct a scale covariant kernel as the outer product of a single window function with the scaling property and that the corresponding distribution is scale covariant as well as being computationally efficient and having reduced interference.

\section{Background on Kernel Decomposition}

Real-valued, bounded discrete TFDs are specified by a conjugate symmetric discrete kernel $\psi(n, m)$ in the time-lag domain and can be expressed in an inner product form using the transformation $\left(n_{1}=n+m / 2, n_{2}=n-m / 2\right)$ on the 
variables $(n, m)$. This corresponds to a $45^{\circ}$ clockwise rotation of $\psi(n, m)$, and the time-frequency representation is given by [7]

$$
\begin{aligned}
& C(n, \omega ; \psi) \\
& \begin{array}{l}
=\sum_{n_{1}=-N}^{N} \sum_{n_{2}=-N}^{N}\left[s\left(n+n_{1}\right) e^{-j \omega\left(n+n_{1}\right)}\right] \psi\left(-\frac{n_{1}+n_{2}}{2}, n_{1}-n_{2}\right) \\
\quad \times\left[s\left(n+n_{2}\right) e^{-j \omega\left(n+n_{2}\right)}\right]^{*} \\
=\left\langle\tilde{\Psi} S_{-n} M_{-\omega} \mathbf{s}, S_{-n} M_{-\omega} \mathbf{s}\right\rangle,
\end{array}
\end{aligned}
$$

where $S_{-n} s\left(n_{1}\right)=s\left(n_{1}+n\right)$ and $M_{-\omega} s\left(n_{1}\right)=s\left(n_{1}\right) e^{-j \omega n_{1}}$ are the time- and frequency-shift operators on $l_{2}$, respectively, and $\tilde{\boldsymbol{\Psi}}$ is a self-adjoint, bounded linear operator on $l_{2}$, which depends on the discrete kernel $\psi(n, m)$. Since the discrete time-frequency kernels are associated with finitedimensional linear operators, they can be represented by matrices,

$$
\begin{gathered}
{[\tilde{\boldsymbol{\Psi}}]_{i j}=\psi\left(\frac{i+j}{2}-N-1, j-i\right)} \\
\text { for } 1 \leq i \leq 2 N+1, \quad 1 \leq j \leq 2 N+1,
\end{gathered}
$$

where $\tilde{\boldsymbol{\Psi}}$ operates on the $2 N+1$-length signal vector $\mathbf{s}=$ $\left[\begin{array}{lllll}s(-N) & s(-N+1) & \cdots & s(N-1) & s(N)\end{array}\right]^{T}$. The matrix $\tilde{\Psi}$ can be written as a weighted sum of the outer products of its eigenvectors, that is, $\widetilde{\Psi}=\sum_{i=1}^{2 N+1} \lambda_{i} \mathbf{e}_{i} \mathbf{e}_{i}^{T}$. It has been shown that since $\tilde{\boldsymbol{\Psi}}$ can be written as a weighted sum of the outer products of its eigenvectors, the corresponding TFD can be written as a sum of weighted spectrograms $[6,8]$. Therefore, any discrete-time TFD corresponding to a $2 N+1 \times 2 N+1$ kernel matrix $\tilde{\boldsymbol{\Psi}}$ can be written as

$$
C(n, \omega ; \psi)=\sum_{k=1}^{2 N+1} \lambda_{k} \operatorname{SP}\left(n, \omega ; \mathbf{e}_{k}\right),
$$

where $\operatorname{SP}\left(n, \omega ; \mathbf{e}_{k}\right)=\left|\left\langle S_{-n} M_{-\omega} s, \mathbf{e}_{k}\right\rangle\right|^{2}=\mid \sum_{n_{1}=-N}^{N} s(n+$ $\left.n_{1}\right)\left.e^{-j \omega\left(n+n_{1}\right)} e_{k}^{*}\left(n_{1}\right)\right|^{2}$ is the spectrogram of the signal computed with the $k$ th eigenvector $\mathbf{e}_{k}$ as the window function, and $\lambda_{k} \mathrm{~s}$ are the corresponding eigenvalues used to weight each spectrogram. For the case where $\tilde{\boldsymbol{\Psi}}$ is a $(2 N+1) \times(2 N+1)$ matrix, the fast algorithms consist of using low-dimensional approximations to $\tilde{\Psi}$, that is, only the largest magnitude eigenvalues are used, such that only a few spectrograms are needed for the evaluation. The full sum evaluates the generalized discrete-time TFD by calculating the weighted sum of $2 N+1$ spectrograms. This eigendecomposition approach is also valid for continuous-time TFDs as discussed in [6].

2.1. Modified Eigendecomposition. We have recently shown that the eigendecomposition of time-frequency kernels can be further simplified by taking the centrosymmetric structure of the kernels into account [5]. Instead of doing a full eigendecomposition of the kernel, the decomposition is done on a submatrix, and the whole kernel is reconstructed in terms of the eigenvectors of this submatrix along with an impulse function. This approach reduces the computational complexity by considering smaller size matrices and represents the TFD in terms of short-time Fourier transforms (STFTs) that are less computationally complex.

Let $\tilde{\boldsymbol{\Psi}}$ be the centrosymmetric kernel matrix corresponding to a TFD that satisfies the time marginal and the timesupport properties. This centrosymmetric matrix can be written in terms of submatrices and vectors as

$$
\tilde{\boldsymbol{\Psi}}=\left[\begin{array}{ccc}
\mathbf{0}_{N \times N} & \mathbf{z} & \mathbf{B} \\
\mathbf{z}^{T} & 1 & \mathbf{z}^{T} \mathbf{J} \\
\mathbf{J B J} & \mathbf{J z} & \mathbf{0}_{N \times N}
\end{array}\right],
$$

where $\mathbf{B}$ is an $N \times N$ lower triangular matrix, $\mathbf{J}$ is the $N \times N$ symmetric elementary matrix defined as $J_{i, j}=\delta_{i, N-j+1}$, for $1 \leq i, j \leq N$, with $\delta_{i, j}$ being the Kronecker delta, and $\mathbf{z}$ is an $N \times 1$ vector. For example, for the Born-Jordan kernel of size $5 \times 5$

$$
\tilde{\Psi}=\left[\begin{array}{ccccc}
0 & 0 & \frac{1}{3} & 0 & 0 \\
0 & 0 & \frac{1}{2} & \frac{1}{3} & 0 \\
\frac{1}{3} & \frac{1}{2} & 1 & \frac{1}{2} & \frac{1}{3} \\
0 & \frac{1}{3} & \frac{1}{2} & 0 & 0 \\
0 & 0 & \frac{1}{3} & 0 & 0
\end{array}\right], \quad \mathbf{B}=\left[\begin{array}{cc}
0 & 0 \\
\frac{1}{3} & 0
\end{array}\right], \quad \mathbf{z}=\left[\begin{array}{c}
\frac{1}{3} \\
\frac{1}{2}
\end{array}\right]
$$

The modified kernel decomposition algorithm described in [5] can be summarized as follows (readers who are interested in the details are referred to [5]):

Step 1. Obtain the upper right $N+1 \times N+1$ submatrix of $\tilde{\boldsymbol{\Psi}}$, called $\mathbf{R}$, where

$$
\mathbf{R}=\left[\begin{array}{cc}
\mathbf{z} & \mathbf{B} \\
1 & \mathbf{z}^{T} \mathbf{J}
\end{array}\right]
$$

Step 2. Do an eigendecomposition on JB, the rotated version of submatrix $\mathbf{B}$, to obtain the eigenvectors $\mathbf{J} \mathbf{x}_{i}$ with eigenvalues $-\lambda_{i}$, where $\mathbf{x}_{i}$ s are the eigenvectors of the matrix, - BJ [9].

Step 3. Compute STFTs with zero-padded versions of the impulse function $(\delta), \mathbf{z}, \mathbf{J} \mathbf{x}_{i}$, and $\mathbf{x}_{i}$. Combine the crossspectrograms computed using the different window pairs to construct the TFD as follows:

$$
\begin{aligned}
C(n, \omega ; \psi)= & \sum_{i=1}^{N}-4 \lambda_{i} \operatorname{Re}\left\{\operatorname{SP}_{\widetilde{\mathbf{x}}_{i}, \tilde{\mathbf{x}}_{i}}(n, \omega)\right\}+2 \operatorname{Re}\left\{\operatorname{SP}_{\widetilde{\mathbf{z}}, \tilde{\delta}}(n, \omega)\right\} \\
& +2 \operatorname{Re}\left\{\operatorname{SP}_{\tilde{\mathbf{z}}, \tilde{\delta}}(n, \omega)\right\}+\operatorname{SP}_{\widetilde{\delta}, \tilde{\delta}}(n, \omega),
\end{aligned}
$$

where $(\because)$ refers to the zero-padded versions of the original vectors by $N+1$ points, and the cross-spectrogram is defined as $\operatorname{SP}_{\widetilde{\mathbf{x}}_{i}, \widetilde{\mathbf{x}}_{i}}(n, \omega)=\operatorname{STFT}_{\widetilde{x}_{i}}(n, \omega) \operatorname{STFT}_{J \widetilde{x}_{i}}^{*}(n, \omega)$. 


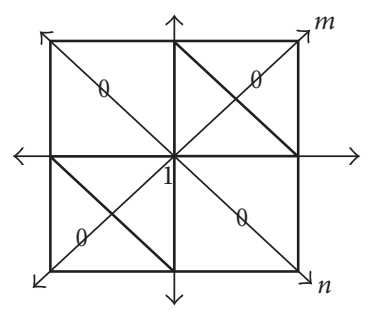

(a)

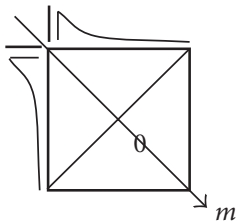

(b)

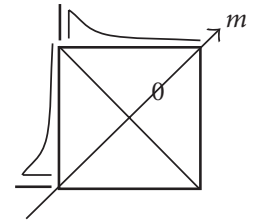

(c)
Figure 1: (a) 45-degree tilted form of the kernel with zeros filled in to form the centrosymmetric matrix $\tilde{\boldsymbol{\Psi}}$, (b) eigensystem decomposition of 90-degree rotated right upper quadrant of the kernel matrix, corresponding to matrix $\mathbf{R}$, with cartoon eigenvectors and the impulse function (Step 2 in the algorithm), and (c) synthesis by rotating back corresponding to Step 3 in the algorithm.

The modified eigendecomposition described above is illustrated in Figure 1. This modified eigendecomposition approach reduces the computational complexity by reducing the number of spectrograms and using windows that are shorter in length. In this paper, this approach will be used to construct computationally efficient scale covariant kernels.

\section{Scale Covariant Computationally Efficient TFDs}

The eigendecomposition algorithm described in the previous section shows that the computation of any TFD can be reduced to the computation of cross-spectrograms using the eigenvectors of the kernel submatrix as the window functions. In order to construct a scale covariant timefrequency kernel, we first have to determine the corresponding eigenvectors. In this section, we will determine whether the eigenvectors of a scale covariant kernel have any particular structure.

In order for the TFD to be scale covariant, the crossspectrograms that constitute the TFD should each be scale covariant. This is a sufficient but not necessary condition. The cross-spectrograms will be scale covariant as long as the STFTs involved are scale covariant. Therefore, we want to determine the eigenvectors such that the STFTs computed using them as window functions are scale covariant. First, we will find a condition on the eigenvectors such that the corresponding STFTs are scale covariant. Then, we will construct kernels using these eigenvectors. The following proofs will be given in the continuous time domain since exact scale covariance cannot be achieved in discrete-time [10]. The results will then be extended for discrete-time for purposes of implementation.
Theorem 1. The Short-Time Fourier Transform of a signal is scale covariant if and only if the window function satisfies the scale invariance property, that is, $h(a t)=(1 / \sqrt{a}) h(t)$ for $a \in$ $R$.

Proof. If the window function is scale invariant, then for a scaled signal $\sqrt{a} s(a t)$, the STFT is

$$
\begin{aligned}
\operatorname{STFT}_{\text {scaled }}(t, \omega) & =\int h(t-\tau) \sqrt{a} s(a \tau) e^{-j \omega \tau} d \tau \\
& =\frac{1}{\sqrt{a}} \int h\left(t-\frac{\tau^{\prime}}{a}\right) s\left(\tau^{\prime}\right) e^{-j \omega \tau^{\prime} / a} d \tau^{\prime} \\
& =\frac{1}{\sqrt{a}} \int h\left(\frac{a t-\tau^{\prime}}{a}\right) s\left(\tau^{\prime}\right) e^{-j \omega \tau^{\prime} / a} d \tau^{\prime} \\
& =\int h\left(a t-\tau^{\prime}\right) s\left(\tau^{\prime}\right) e^{-j \omega \tau^{\prime} / a} d \tau^{\prime} \\
& =\operatorname{STFT}\left(a t, \frac{\omega}{a}\right),
\end{aligned}
$$

where the second to last equality is obtained by the scale invariance property of the window function $h$. The converse can be proven in a similar manner.

Now that we have shown that scale invariant window functions produce scale covariant STFTs, we have to determine the class of window functions that satisfy this scale invariance property.

Theorem 2. The only real function $h(t)$ which satisfies the scale invariance property is $K / \sqrt{t}$, where $K \in R$.

Proof. In order to find $h(t)$ such that $h(a t)=(1 / \sqrt{a}) h(t)$, we use the scale transform which is defined as [1]

$$
D(c)=\frac{1}{\sqrt{2 \pi}} \int_{0}^{\infty} h(t) \frac{e^{-j c \ln t}}{\sqrt{t}} d t .
$$

If we take the scale transform of the two sides in the equality $h(a t)=(1 / \sqrt{a}) h(t)$, we obtain

$$
\begin{gathered}
\frac{1}{\sqrt{2 \pi}} \int_{0}^{\infty} h(a t) \frac{e^{-j c \ln t}}{\sqrt{t}} d t=\frac{1}{\sqrt{a}} D(c), \\
\frac{1}{\sqrt{2 \pi}} \frac{1}{\sqrt{a}} e^{j c \ln a} \int_{0}^{\infty} h\left(t^{\prime}\right) \frac{e^{-j c \ln t^{\prime}}}{\sqrt{t^{\prime}}} d t^{\prime}=\frac{1}{\sqrt{a}} D(c), \\
e^{j c \ln a} D(c)=D(c) .
\end{gathered}
$$

The last equality is true when $c=0$. This implies that $D(c)=0$, for $c \neq 0$. Therefore, the only real solution for $D(c)$ is $K \delta(c), K \in R$. By taking the inverse scale transform of $K \delta(c)$, we can obtain $h(t)$ as follows:

$$
h(t)=\int K \delta(c) \frac{e^{j c \ln t}}{\sqrt{t}} d c=\frac{K}{\sqrt{t}}, \quad t>0 .
$$

3.1. Construction of a Scale Covariant Kernel. The discretetime equivalent of the scale invariant function $K / \sqrt{t}$ is $K / \sqrt{n}$ for $n \geq 1$. In order for the TFD computed by (8) to be scale covariant, all of the window functions used in computing the spectrograms should be of the form $K / \sqrt{n}$. This implies 
that the eigenvectors $\mathbf{J x}_{\mathbf{i}}$ and the vector $\mathbf{z}$ in (8) should all be in this form. Since the vectors of the form $K / \sqrt{n}$ are linearly dependent, the kernel submatrix JB can have at most one eigenvector Jx. Therefore, the scale covariant kernel is constructed using a single eigenvector. We can generate a family of scale covariant kernels using different values for $K$.

In the following discussions, we will choose $z(n)=$ $K_{1} / \sqrt{n}$ and $J x(n)=K_{2} / \sqrt{n}$. A $2 N+1 \times 2 N+1$ kernel, $\tilde{\Psi}$, can be constructed using these two vectors based on (5) as follows:

$$
\tilde{\Psi}=\left[\begin{array}{ccccccc}
\ddots & 0 & 0 & \vdots & \vdots & \vdots & \vdots \\
\cdots & \cdots & 0 & \frac{K_{1}}{\sqrt{2}} & \frac{K_{2}^{2}}{\sqrt{2}} & \frac{K_{2}^{2}}{2} & \cdots \\
\cdots & \cdots & 0 & K_{1} & K_{2}^{2} & \frac{K_{2}^{2}}{\sqrt{2}} & \cdots \\
\cdots & \frac{K_{1}}{\sqrt{2}} & K_{1} & 1 & K_{1} & \frac{K_{1}}{\sqrt{2}} & \cdots \\
\cdots & \frac{K_{2}^{2}}{\sqrt{2}} & K_{2}^{2} & K_{1} & 0 & \cdots & \cdots \\
\cdots & \frac{K_{2}^{2}}{2} & \frac{K_{2}^{2}}{\sqrt{2}} & \frac{K_{1}}{\sqrt{2}} & 0 & \cdots & \cdots \\
\vdots & \vdots & \vdots & \vdots & 0 & 0 & \ddots
\end{array}\right]
$$

The only eigenvector of this matrix is $\left[\begin{array}{llll}1 & 1 / \sqrt{2} & 1 / \sqrt{3} & 1 / 2\end{array}\right]^{T}$ which is the scale invariant window function $x(n)=$ $1 / \sqrt{n}$. Defining $\tilde{\delta}=\left[\begin{array}{lll}\mathbf{0}_{1 \times N} & 1 & \mathbf{0}_{1 \times N}\end{array}\right], \tilde{\mathbf{x}}=\left[\begin{array}{ll}\mathbf{0}_{1 \times N+1} & \mathbf{x}\end{array}\right]$, the corresponding time-frequency distribution can be written as follows:

$$
\begin{aligned}
& \operatorname{TFD}(n, \omega)=\operatorname{SP}_{\tilde{\mathbf{z}}, \tilde{\delta}}(n, \omega)+\operatorname{SP}_{\tilde{\delta}, \tilde{\mathbf{z}}}(n, \omega) \\
& +\mathrm{SP}_{\mathbf{J} \tilde{\mathbf{z}}, \tilde{\delta}}(n, \omega)+\mathrm{SP}_{\widetilde{\delta}, \widetilde{\mathbf{z}}}(n, \omega) \\
& +\mathrm{SP}_{\tilde{\mathbf{x}}, \tilde{\mathbf{X}}}(n, \omega)+\mathrm{SP}_{\mathbf{J}_{\tilde{\mathbf{x}}, \tilde{\mathbf{x}}}}(n, \omega)+\mathrm{SP}_{\widetilde{\delta}, \tilde{\delta}}(n, \omega) \\
& =2 \operatorname{Re}\left\{\operatorname{SP}_{\widetilde{\mathbf{x}}, \tilde{\mathbf{x}}}(n, \omega)\right\}+2 \operatorname{Re}\left\{\operatorname{SP}_{\widetilde{\mathbf{z}}, \tilde{\delta}}(n, \omega)\right\} \\
& +2 \operatorname{Re}\left\{\operatorname{SP}_{\mathbf{J} \tilde{\mathbf{z}}, \tilde{\delta}}(n, \omega)\right\}+\operatorname{SP}_{\tilde{\delta}, \tilde{\delta}}(n, \omega),
\end{aligned}
$$

where SP corresponds to the cross-spectrogram using the different window functions. Note that for this scale covariant kernel, the vector $\mathbf{z}$ is the same as $\mathbf{J x}$ up to a scalar constant. Therefore, this representation relies on the computation of one nontrivial STFT, the one computed using $\tilde{\mathbf{x}}$ as the window function. The other STFTs are trivial as in the case of the impulse function or can be easily obtained from this STFT by applying time reversal and amplitude scaling operations.

3.2. Computational Complexity. The computational complexity of this time-frequency distribution consists of computing 3 distinct STFTs, one of which is trivial. Based on the results in [5], the total number of real multiplications per time-frequency point for a TFD with size $2 N+1 \times N_{F}$ is given as follows:

$$
3+\frac{3\left[N+(N / 2) \log _{2}\left(N_{F} / 2\right)+(2 / 3) N_{F}\right]}{N_{F}}+4,
$$

where the first term corresponds to the 3 real multiplications required for computing the spectrogram with the impulse function; the second term corresponds to the computational complexity of the cross-spectrograms computed using $\mathbf{J x}$ and $\mathbf{x}$, each of which requires $N$ complex multiplications between the window and the signal, an $N$ point FFT, and $2 N_{F}$ real multiplications for multiplying with the complex conjugate; the third term corresponds to the computational complexity of multiplying the STFTs computed using the impulse function and $\mathbf{J} \tilde{\mathbf{z}}$ or $\tilde{\mathbf{z}}$. Therefore, the total computational complexity is in the order of $O(\log N)$ compared to $O\left(N^{2} \log N\right)$ for bilinear time-frequency distributions belonging to Cohen's class with a nontrivial kernel function, that is, excluding the Wigner distribution.

3.3. Properties. In this section, some properties of the proposed scale covariant, computationally efficient kernel will be discussed.

(1) Time Marginal. This kernel will satisfy the time marginal since $\psi(n, 0)=\delta(n)$ by construction (see $(13))$.

(2) Frequency Marginal. This kernel will not satisfy the frequency marginal since

$$
\sum_{n} \psi(n, m) \neq 1 \quad \forall m
$$

(3) Reduced Interference Distribution. Reduced interference is a desired property for bilinear time-frequency distributions especially in the case of multicomponent signals where cross-terms contaminate the representation. Reduced interference distribution requires the kernel $\phi(\theta, m)$ to decay as we move away from the $\theta, m$ axis. This condition implies that the kernel is smoothly decaying, that is, $\psi(n, m)$ should also be smoothly decaying as $n$ increases. In our case, the kernel is given by

$$
\psi(n, m)= \begin{cases}1, & n=0, m=0 \\ \frac{K_{2}^{2}}{\sqrt{|n+m / 2|} \sqrt{|n-m / 2|}}, & |n|<\frac{|m|}{2}, \\ \frac{K_{1}}{\sqrt{|n|+|m| / 2}}, & |n|=\frac{|m|}{2},\end{cases}
$$

which decays as a function of $n$.

\section{Experimental Results}

In this section, two examples will be given to illustrate the scale covariance and reduced interference properties of the proposed distribution.

4.1. Example 1: Evaluation of the Scale Covariance. Let $s$ be a signal that consists of the sum of two Gabor logons, where the Gabor logons are the time-shifted and -scaled versions of 


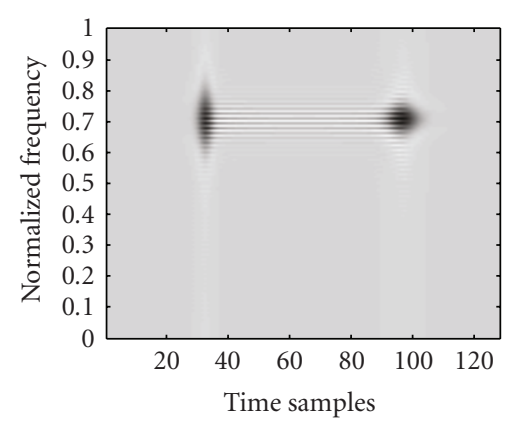

(a)

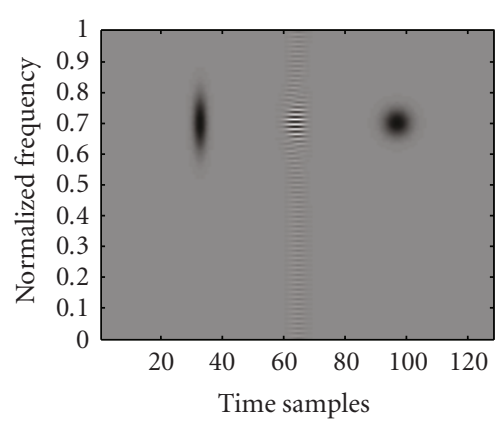

(b)

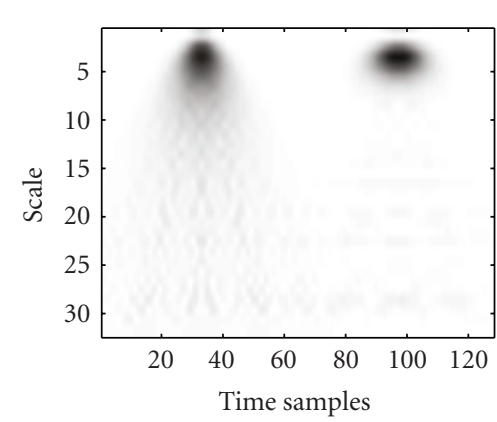

(c)

FIgURE 2: Scale covariant distribution for a Gabor logon and its time-scaled version. (a) Scale covariant distribution, (b) Wigner distribution, and (c) Wavelet distribution using the Morlet wavelet.

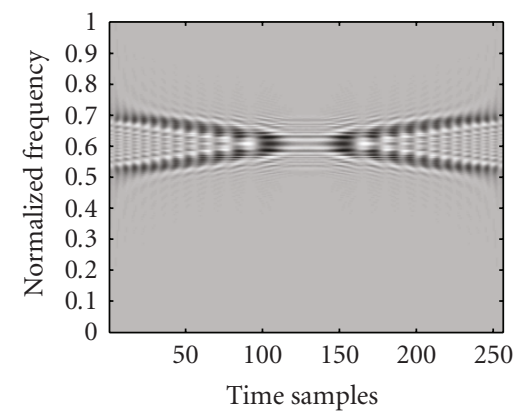

(a)

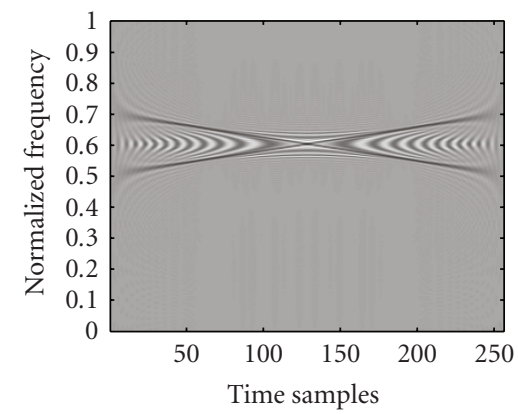

(b)

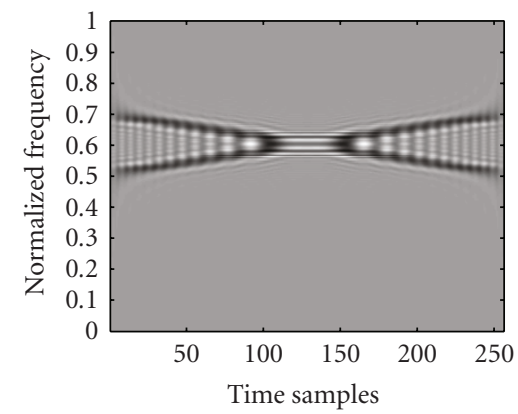

(c)

Figure 3: Time-frequency distribution for two crossing chirp signals. (a) Scale covariant distribution, (b) Wigner distribution, and (c) Reduced interference distribution using the Born-Jordan kernel.

each other, that is, $s(n)=\exp \left(-\left(n-n_{0}\right)^{2} / \sigma\right) \exp (-j 2 \pi(n-$ $\left.\left.n_{0}\right) k_{0}\right)+\exp \left(-\left(n-n_{1}\right)^{2} / a \sigma\right) \exp \left(-j 2 \pi\left(n-n_{1}\right) k_{0}\right)$. The timefrequency kernel is constructed as described in the previous section using $K_{1}=K_{2}=0.5$, that is, $z(n)=J x(n)=1 / 2 \sqrt{n}$. Figure 2 compares the time-frequency representations of this signal for three different distributions: the computational efficient scale covariant distribution introduced in this paper, the Wigner distribution, and the wavelet transform using the Morlet wavelet. It can be seen that the Wigner distribution and the scale covariant distribution are similar to each other. Both of these representations scale in time and frequency according to the time scaling factor $a$, with the difference being the resolution. Wigner distribution achieves higher resolution than the scale covariant kernel since it is based on computing cross-spectrograms using multiple windows rather than a single window. When the scale covariant distribution is compared to the wavelet transform, it can be seen that wavelet transform scales in time and frequency at the expense of reduced resolution. This reduced resolution is due to the fact that the wavelet transform has constant$\mathrm{Q}$ resolution, that is, at low frequencies it provides highfrequency resolution and low-time resolution, whereas at high frequencies the opposite is true. Therefore, the class of scale covariant distributions proposed in this paper satisfies several desired properties such as high time-frequency resolution, scale covariance, and low computational complexity, simultaneously.
4.2. Example 2: Evaluation of the Cross-Terms. In this example, we compare the proposed scale covariant timefrequency distribution to the Wigner distribution and a reduced interference distribution (Born-Jordan distribution) in terms of the amount of cross-terms. For this purpose, we consider the sum of two chirp signals, $x(t)=\exp \left(j\left(\omega_{1} t+\right.\right.$ $\left.\left.\beta_{1} t^{2}\right)\right)+\exp \left(j\left(\omega_{2} t+\beta_{2} t^{2}\right)\right)$, where $\omega_{2}=\omega_{1}+\beta_{1} t_{\text {final }}$ and $\beta_{2}=$ $-\beta_{1}$. The time-frequency kernel is constructed as described in the previous example using $K_{1}=K_{2}=0.5$, that is, $z(n)=$ $J x(n)=1 / 2 \sqrt{n}$. The three time-frequency distributions obtained using the three different kernel functions are shown in Figure 3. We quantify the amount of cross-terms in the different TFDs using a signal-to-noise ratio (SNR) type measure in the time-frequency plane as follows:

$$
\mathrm{SNR}=10 \log \frac{\sum_{n} \sum_{k} \mathrm{TFD}_{\text {auto-terms }}^{2}(n, k)}{\sum_{n} \sum_{k} \mathrm{TFD}_{\text {cross-terms }}^{2}(n, k)},
$$

which measures the ratio of the energy of the autoterms to the energy of the cross-terms. The SNR for the three distributions are $0.0363 \mathrm{~dB}$ for Wigner distribution, $2.6643 \mathrm{~dB}$ for scale covariant distribution, and $3.1528 \mathrm{~dB}$ for Born-Jordan distribution. As we can see from the actual distributions and the SNRs, the proposed scale covariant distribution is better in terms of suppressing the cross-terms compared to the Wigner distribution. The proposed distribution also has lower computational complexity than Born-Jordan 
distribution at the expense of a slight increase in the energy of the cross-terms. Therefore, the proposed distribution achieves a tradeoff between the Wigner distribution and the general class of reduced interference distributions in terms of computational complexity and the amount of cross-terms.

\section{Conclusions}

In this paper, we introduced a new method for constructing scale covariant time-frequency distributions. It is shown that the scale covariant kernels can be constructed using a single scale invariant eigenvector combined with the impulse function. This realization leads to a computationally efficient TFD computation algorithm where the TFD is composed of three short-time Fourier transforms, of which only one is nontrivial and distinct. The resulting distribution is shown to be scale covariant. Comparisons with other well-known scale covariant representations show that the proposed distribution achieves scale covariance simultaneously with low computational complexity, high-frequency resolution, and reduced interference.

\section{References}

[1] L. Cohen, Time-Frequency Analysis, Prentice-Hall, Englewood Cliffs, NJ, USA, 1995.

[2] C. S. Burrus, R. A. Gopinath, and H. Guo, Introduction to Wavelets and Wavelet Transforms, Prentice-Hall, Englewood Cliffs, NJ, USA, 1997.

[3] J. Bertrand and P. Bertrand, "A class of affine Wigner functions with extended covariance properties," Journal of Mathematical Physics, vol. 33, no. 7, pp. 2515-2527, 1992.

[4] A. Papandreou, F. Hlawatsch, and G. F. BoudreauxBartels, "The hyperbolic class of quadratic time-frequency representations-part I: constant-Q warping, the hyperbolic paradigm, properties, and members," IEEE Transactions on Signal Processing, vol. 41, no. 12, pp. 3425-3444, 1993.

[5] S. Aviyente and W. J. Williams, "A centrosymmetric kernel decomposition for time-frequency distribution computation," IEEE Transactions on Signal Processing, vol. 52, no. 6, pp. 15741584, 2004.

[6] G. S. Cunnigham and W. J. Williams, "Kernel decomposition of time-frequency distributions," IEEE Transactions on Signal Processing, vol. 42, no. 6, pp. 1425-1442, 1994.

[7] J. Jeong and W. J. Williams, "Alias-free generalized discretetime time-frequency distributions," IEEE Transactions on Signal Processing, vol. 40, no. 11, pp. 2757-2765, 1992.

[8] M. G. Amin, "Spectral decomposition of time-frequency distribution kernels," IEEE Transactions on Signal Processing, vol. 42, no. 5, pp. 1156-1165, 1994.

[9] A. Cantoni and P. Butler, "Eigenvalues and eigenvectors of symmetric centrosymmetric matrices," Linear Algebra and Its Applications, vol. 13, no. 3, pp. 275-288, 1976.

[10] E. J. Zalubas, Signal processing and pattern recognition in scale-content domains: theory and applications, Ph.D. thesis, University of Michigan, Ann Arbor, Mich, USA, 1999. 